\title{
TRICLOBS Portable Triband Color Lowlight Observation System
}

\author{
Alexander Toet*, Maarten A. Hogervorst \\ TNO Human Factors, P.O. Box 23, 3769 ZG Soesterberg, the Netherlands
}

\begin{abstract}
We present the design and first test results of the TRICLOBS (TRI-band Color Low-light OBServation) system The TRICLOBS is an all-day all-weather surveillance and navigation tool. Its sensor suite consists of two digital image intensifiers (Photonis ICU's) and an uncooled longwave infrared microbolometer (XenICS Gobi 384). The night vision sensor suite registers the visual $(400-700 \mathrm{~nm})$, the near-infrared $(700-1000 \mathrm{~nm})$ and the longwave infrared $(8-14 \mu \mathrm{m})$ bands of the electromagnetic spectrum. The optical axes of the three cameras are aligned, using two dichroic beam splitters: an ITO filter to reflect the LWIR part of the incoming radiation into the thermal camera, and a B43-958 hot mirror to split the transmitted radiation into a visual and NIR part. The individual images can be monitored through two LCD displays. The TRICLOBS provides both digital and analog video output. The digital video signals can be transmitted to an external processing unit through an Ethernet connection. The analog video signals can be digitized and stored on on-board harddisks. An external processor is deployed to apply a fast lookup-table based color transform (the Color-the-Night color mapping principle) to represent the TRICLOBS image in natural daylight colors (using information in the visual and NIR bands) and to maximize the detectability of thermal targets (using the LWIR signal). The external processor can also be used to enhance the quality of all individual sensor signals, e.g. through noise reduction and contrast enhancement.
\end{abstract}

Keywords: image fusion, false color, natural color mapping, real-time fusion, lookup tables

\section{INTRODUCTION}

Night vision cameras are a vital source of information for a wide-range of critical military and law enforcement applications related to surveillance, reconnaissance, intelligence gathering, and security. The two most common nighttime imaging systems cameras are low-light-level (e.g., image-intensified) cameras, which amplify the reflected visible to near infrared (VNIR) light, and thermal infrared (IR) cameras, which convert invisible thermal energy from the midwave ( 3 to 5 microns) or the long wave ( 8 to 12 microns) part of the spectrum into a visible image. Until recently a gray- or greenscale representation of nightvision imagery has been the standard. However, the increasing availability of fused and multi-band infrared and visual nightvision systems has led to a growing interest in the color display of night vision imagery ${ }^{5,7,8,8,12,18,18}$. In principle, color imagery has several benefits over monochrome imagery for surveillance, reconnaissance, and security applications. For instance, color may improve feature contrast, which allows for better scene recognition and object detection. When sensors operate outside the visible waveband, artificial color mappings generally produce false color images whose chromatic characteristics do not correspond in any intuitive or obvious way to those of a scene viewed under natural photopic illumination. This type of false color imagery may disrupt the recognition process, resulting in an observer performance that is even worse compared to that obtained with singleband imagery alone ${ }^{9}$. Several different techniques have been proposed to display night-time imagery in natural daylight colors $^{10-13,16,18}$, some of which have been implemented in realtime nightvision systems ${ }^{1,4,14,15,17}$. Most of these techniques are computationally expensive and/or do not achieve color constancy. We recently introduced a new color mapping that displays night-time imagery in natural daytime colors ${ }^{2}$. This technique is simple and fast, and can easily be deployed in realtime. Moreover, it provides stable colorization under variations in scene content ${ }^{2,3}$.

Here we describe the implementation of this new color mapping in the prototype TRICLOBS (TRI-band Color Low-light OBServation) all-day all-weather surveillance and navigation system. The system displays the co-aligned visual, nearinfrared and thermal signals of respectively two image intensifiers and an uncooled microbolometer in full color. A fast lookup-table implementation of the Color-the-Night color mapping transform ${ }^{2}$ is deployed to represent the TRICLOBS image in natural daylight colors (using information in the visual and NIR bands) and to maximize the detectability of thermal targets (using the LWIR signal).

*lex.toet@tno.nl; phone +31-346-356237; fax +31-346-353977; http://lextoet.googlepages.com/

Multisensor, Multisource Information Fusion: Architectures, Algorithms, and Applications 2009, edited by Belur V. Dasarathy, Proc. of SPIE Vol. 7345, 734503 · (C) 2009 SPIE · CCC code: 0277-786X/09/\$18 · doi: 10.1117/12.817526 


\section{COLOR MAPPING}

The principle of the new lookup-table based color mapping technique is explained in detail $\mathrm{in}^{2}$. For the sake of completeness we will now briefly describe this procedure. First, a false color image is constructed by mapping the different bands of a multisensor nightvision system to respectively the R, G, and B channels of an RGB image (set channel B to zero when only 2 bands are available, and use only the first three principal components when the system provides more than 3 bands). Second, transform the false color image thus obtained into an indexed image using a color lookup table containing a set RGB triples (this is a 3D lookup table, which reduces to $2 \mathrm{D}$ when only 2 bands are available). Finally, replace the false color lookup table of the input multiband nightvision image with a new color lookup table that maps the two image bands onto natural colors. The new color lookup-table can be obtained either by applying a statistical transform to the entries of the original lookup-table, or by a procedure that replaces entries of the original lookup-table by their corresponding natural color values. The statistical transform method transfers the first order statistics (mean and standard deviation) of the color distribution of a representative natural color daytime reference image to the false color multiband nighttime image ${ }^{2,11}$. This mapping is usually performed in a perceptually de-correlated color space (e.g. $l \alpha \beta^{6}$ ). The sample-based method deploys a set of corresponding samples from the combination of a multi-band sensor image of a given scene and a registered naturally colored (RGB) daytime reference image of the same scene to derive a color lookup table transform pair that transfers the color characteristics of the natural color reference image to the false color nighttime image ${ }^{2,3}$. For an 8-bit multi-band system providing 3 or more bands the 3D color lookup table contains 256x256x256 entries (for a 2 band system the 2D table contains 256x256 entries). When the color lookup table contains fewer entries, the color mapping is achieved by determining the closest match of the table entries to the observed multi-band sensor values. Once the color transformation has been derived and the pair of color lookup tables that defines the mapping has been created, they can be used in a real-time application. The lookup table transform requires minimal computing power. An additional advantage of the color lookup transform method is that object colors only depend on the multi-band sensor values and are independent of the image content. As a result, objects keep the same color over time when registered with a moving camera.

In the next sections we first describe the overall system design and the components of a prototype portable tri-band realtime nightvision system that deploys the new lookup-table color transform method. Then we will show the results of some preliminary field trials.

\section{SYSTEM DESIGN}

\subsection{Overview}

The TRICLOBS system combines a three-band nightvision sensor suite, consisting of two digital image intensifiers and a thermal (LWIR) camera, in combination with a $3 \mathrm{D}$ digital position information system. The night vision sensor suite is sensitive in the visual $(400-700 \mathrm{~nm})$, the near-infrared $(700-1000 \mathrm{~nm})$ and the longwave infrared $(8-14 \mu \mathrm{m})$ bands of the electromagnetic spectrum. The optical axes of all cameras are aligned.

Figure 1 shows a schematic representation of the layout of the sensors suite and the beam splitters that are deployed to direct the appropriate band of the incoming radiation to each of the individual sensors. The incoming radiation is first split into a longwave (thermal) and a visual+NIR part by a heat reflecting (hot) mirror. The longwave part of the spectrum is reflected into the lens of the XenICs Gobi camera, while the visual+NIR light is transmitted to the combination of the two Photonis ICUs. The two ICUs are mounted under an angle of 90 degrees. A near-infrared reflecting mirror is used to separate the incoming light such that one ICU registers the visual part and the other ICU only detects the NIR part of the incoming radiation. Figure 2 shows a preliminary test setup of this sensor configuration.

The sensor suite and the mirrors are mounted on a common metal base. The whole configuration is placed in an enclosed housing. 


\subsection{Signal Processing}

The TRICLOBS system delivers both analog video and digital signal output. Figure 3 shows the system wiring diagram.

The 16-bit TCP/IP Ethernet interface of the XenICs Gobi 384 is connected to a Netgear Gigabit Ethernet switch. The SDI channels of both Photonis ICU's are also connected to this Netgear hub through SDI/Etherrnet converters. This enables the user to transmit high quality TRICLOBS signals over an Ethernet connection.

The USB ports of both Photonis ICU's are connected to a high-speed 7-port USB 2.0 hub. This enables the user to interface with the ICU's and to adjust their settings or to download and install preferred settings.

All camera signals can either be stored on disk for offline processing, or can be processed online further as follows. Note that each of the following processing steps can selectively be activated or de-activated.

A Pleora frame grabber (www.pleora.com) can be used to digitize the analog video signals of both ICU's (through the $\mathrm{SDI} /$ Ethernet converters) and the digital output of the Gobi 384 camera, and output these signals to the Netgear Gigabit Ethernet switch.

Three Pinnacle Video Transfer Units (www.pinnaclesys.com/PVT) can be deployed to store (a) the analog video signals of all three cameras and (b) the audio signals of two (optional) external microphones, either on 3 internal $320 \mathrm{~Gb}$ harddisks, or on USB memory sticks. The microphones can for instance be positioned on the front and back of the camera suite. The microphone on front can then be used to register relevant audio information from the registered scene, and the second microphone can for instance be used to record spoken annotations.

An internal U-blox EVK-5P Positioning Engine (www.u-blox.com) provides a position and orientation (i.e. sensor location and viewing direction) signal through the high-speed 7-port USB 2.0 hub.

Two external video displays are provided to simultaneously monitor two of the three video signals (either Visual/NIR, Visual/LWIR, or NIR/LWIR).

The Color-the-Night false color mapping is performed on an external processing unit, connected to the TRICLOBS via an Ethernet connection. This can either be a regular laptop (since the operation is efficiently implemented as a lookup table transform only a minimal amount of computation is required to achieve real-time performance), a regular PC (in case portability is not an issue), or a dedicated PC.

The whole system can either run on an internal battery pack, or on $220 \mathrm{~V} \mathrm{AC}$.

Figure 4 shows the layout of the hardware components of the TRICLOBS system. Figure 5 shows the TRICLOB operation panel, containing all input/output connections, the main power switch, and the start/stop button for the internal image recording 


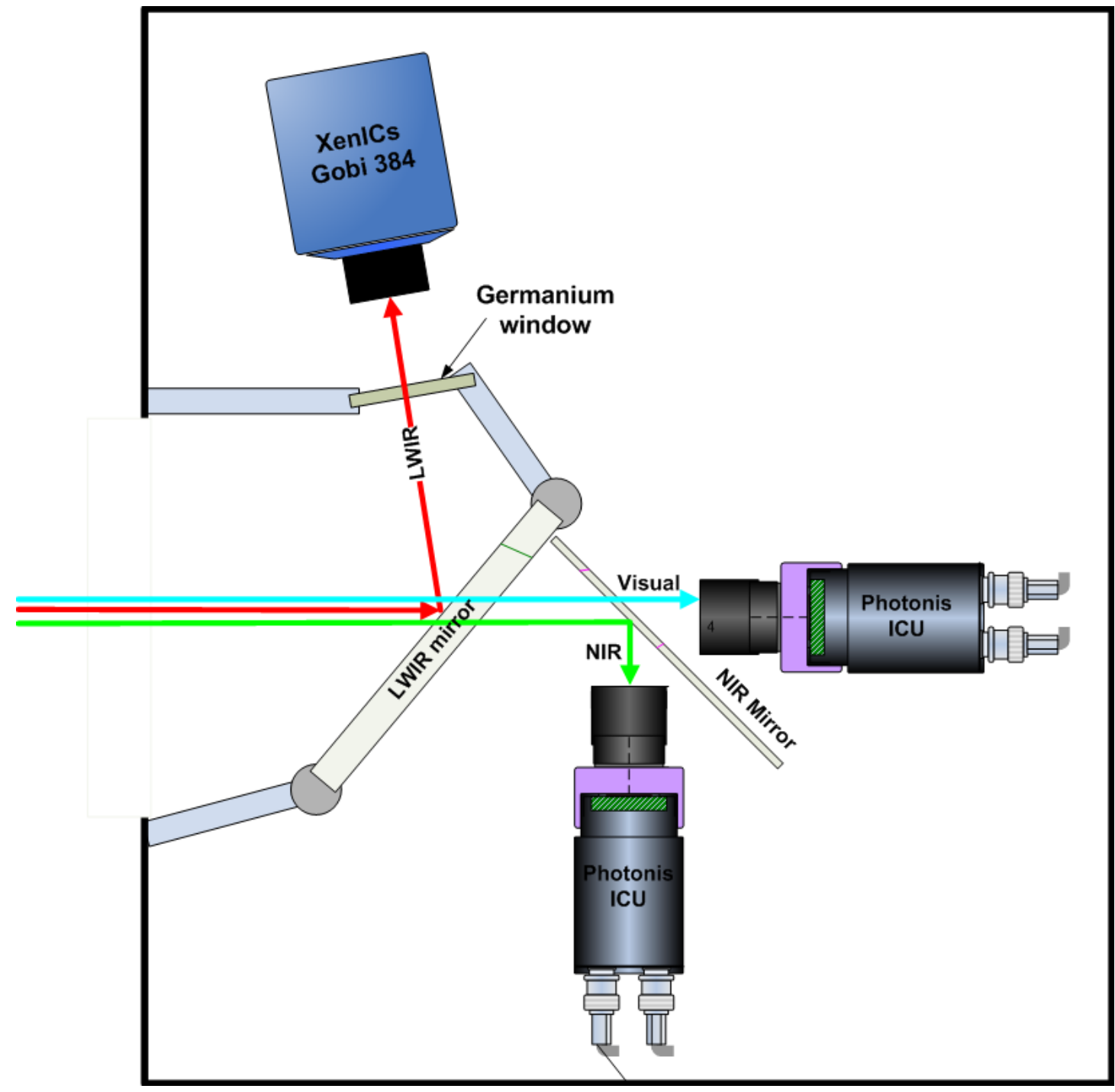

Fig. 1 Schematic layout of the sensors and filters of the TRICLOBS system. 


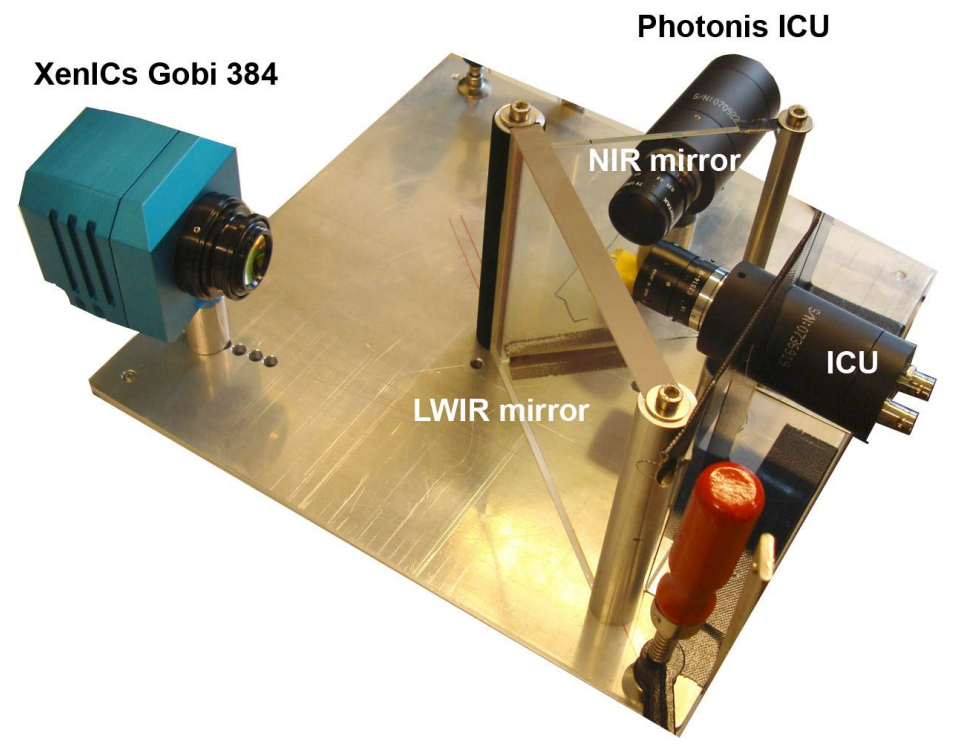

Fig. 2 Preliminary test set-up showing the XenICs Gobi camera and the two Photonis ICU's

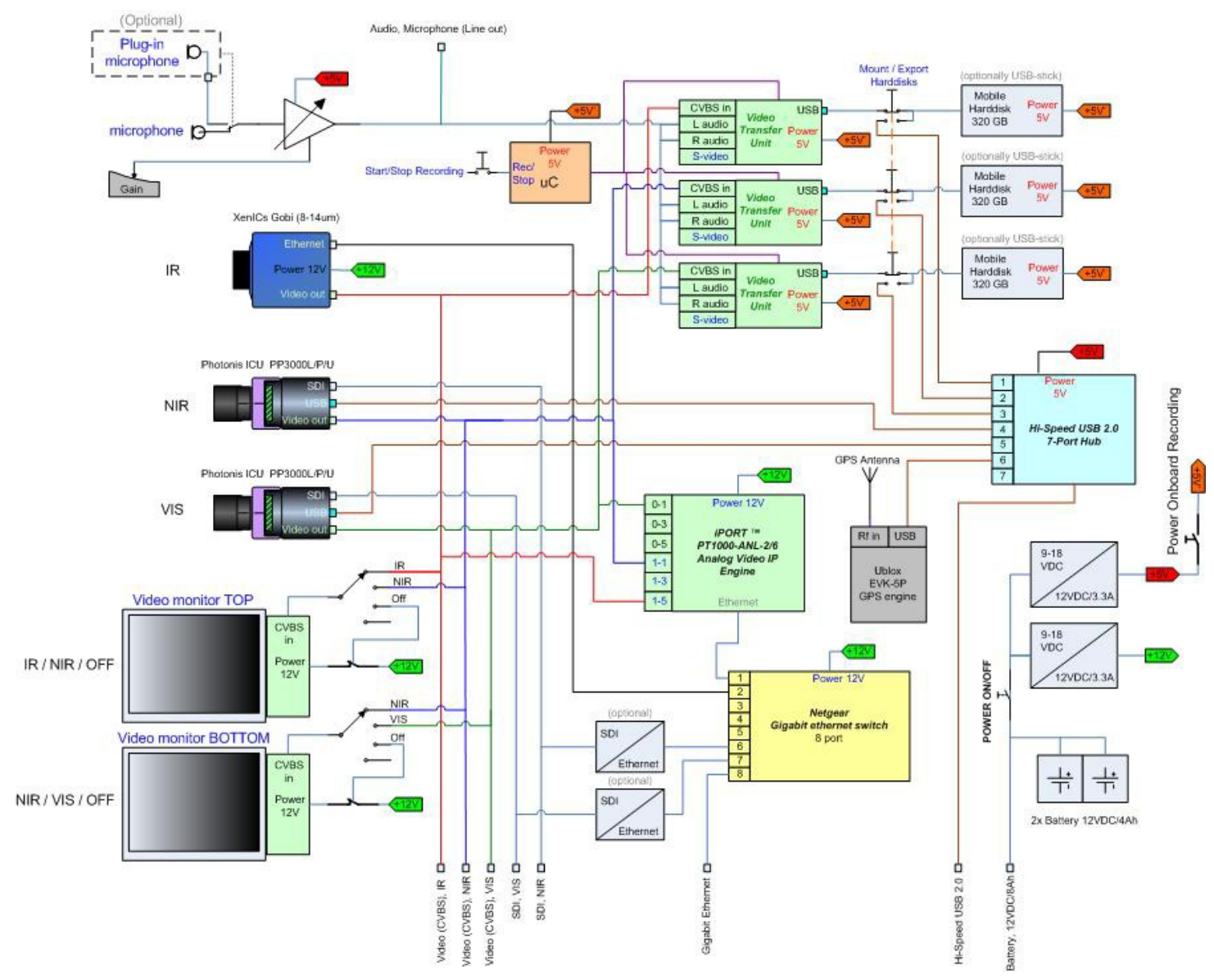

Fig. 3 TRICLOBS wiring scheme. 



Fig. 4 Outside (top) and inside (bottom) top views of the TRICLOBS system 


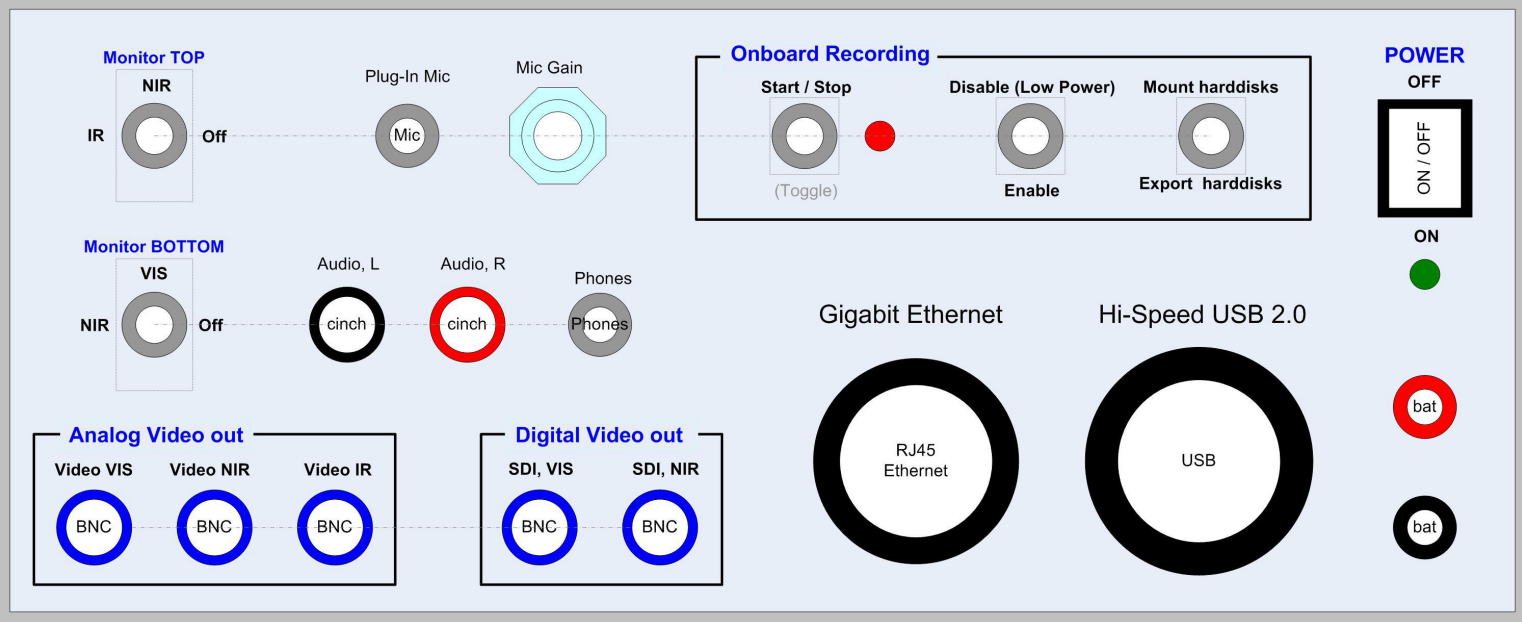

Fig. 5 TRICLOBS operation panel.

\subsection{Digital Image Intensifiers}

The TRICLOBS contains two Photonis Intensified Camera Units (ICUs): an ICU PP3000L and an ICU PP3000U (Fig. 6, see: www.photonis.com). The ICU is a new generation of low light level, intensified CMOS camera. It has a 2/3" CMOS sensor with a spectral response range of 400-900 nm, and delivers both a PAL or NTSC composite video signal output (ITU-R BT.656-4, 640x480 pixels), and an SDI - LVDS $270 \mathrm{Mbits} / \mathrm{s}$ signal. It is equipped with a C-mount lens adapter. Both ICU's are equipped with Pentax C2514M CCTV lenses, with a minimal focal length of $25 \mathrm{~mm}$ and a lens aperture of $\mathrm{F} / 1.4$, resulting in a $\mathrm{FOV}$ of $30.7^{\circ} \times 24.8^{\circ}$.

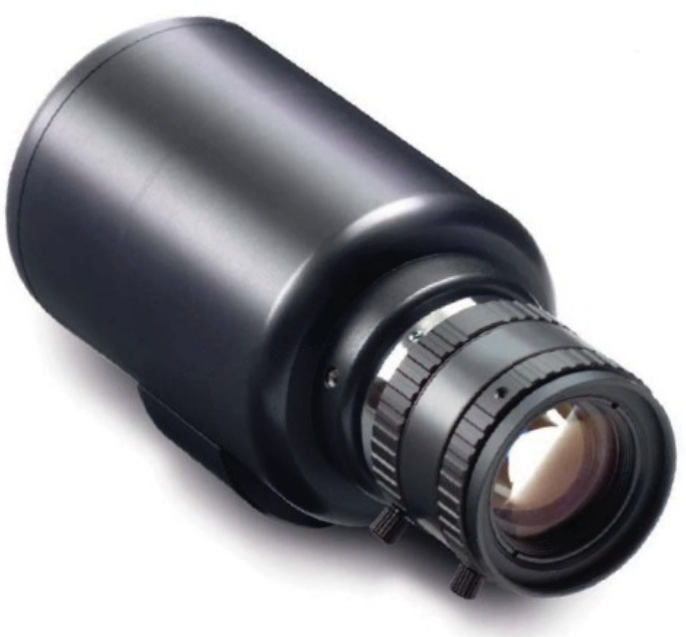

Fig. 6 Photonis Intensified Camera Unit (ICU PP3000L, see: www.photonis.com). 


\subsection{Thermal Camera}

The XenICs Gobi 384 uncooled a-Si infrared microbolometer (Fig. 7, see: www.xenics.com) has a 384 x 288 pixel focal plane array, and a spectral sensitivity range of $8-14 \mu \mathrm{m}$, which is the range of most interest for outdoor applications. It is equipped with an $18 \mathrm{~mm}(\mathrm{f} / 1)$ lens providing a $29.9^{\circ}$ x $22.6^{\circ}$ wide angle view. The Gobi 384 has a 16-bit Ethernet and CameraLink interface.

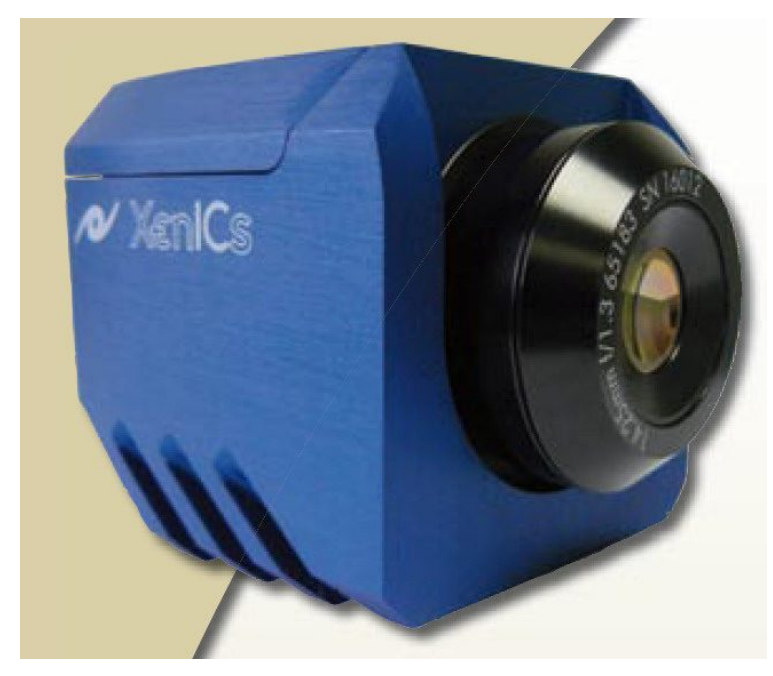

Fig. 7 The XenICs Gobi 384 infrared microbolometer (see: www.xenics.com).

\subsection{LWIR Mirror}

A custom made Melles Griot dichroic beam splitter consisting of Schott N-BK7 Borosilicate Crown glass with an Indium Tin Oxide (ITO) coating (www.ocioptics.com/ito.html) is used to split the LWIR part of the incoming radiation and reflect it into the lens of the XenICs Gobi 384 thermal camera. This filter transmits the visual/near-infrared band (400-900nm) and reflects the longwave (thermal) infrared part (7000-14000nm). According to the specifications provided by Melles Griot (Fig. 8) the reflection R $>84.75 \%$ for $7.5-9.5 \mu \mathrm{m}, \mathrm{R}>87.25 \%$ for $9.5-11.5 \mu \mathrm{m}, \mathrm{R}>90 \%$ for $420-700 \mu \mathrm{m}, \mathrm{R}>80$ for $1064 \mu \mathrm{m}, \mathrm{R}>50 \%$ for $1540-1570 \mu \mathrm{m}$, all measured at an angle of incidence of about $50^{\circ}$.

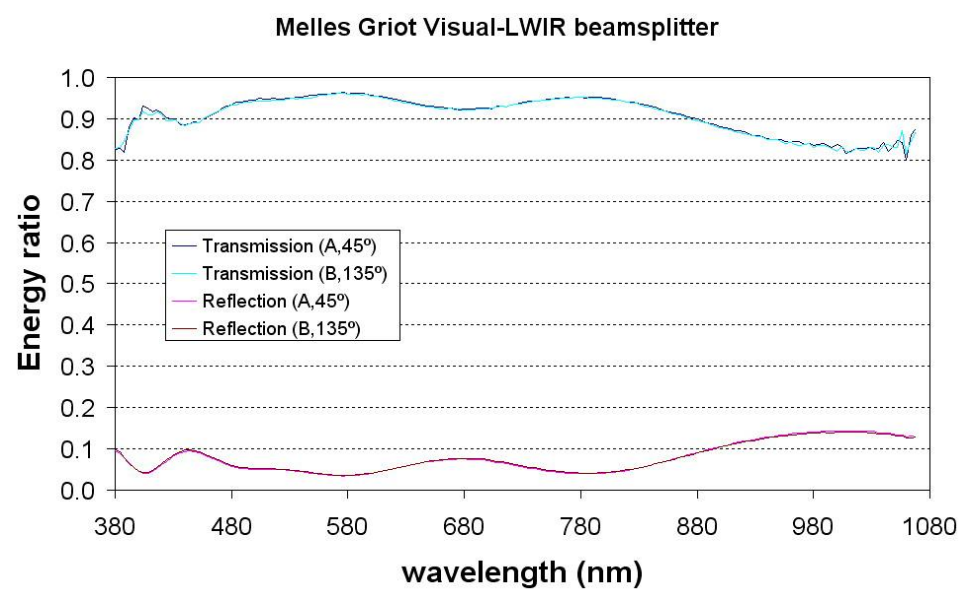

Fig. 8 Spectral transmission of the Melles Griot dichroic beam splitter. 


\subsection{NIR Mirror}

A hot mirror filter (45 deg angle of incidence, type Edmund Optics B43-958, 101x127x3.3 mm, see: www.edmundoptics.com) is deployed to split the visual/near-infrared band $(400-900 \mathrm{~nm})$ by transmitting the visual (400-700nm) and reflecting the NIR part (700-900nm) of the spectrum (Fig. 9).



Fig. 9 (a) Transmission and reflection characteristic of the Edmund Optics B43-958 hot mirror, for a 45 deg angle between the mirror surface and the incoming radiation beam.

\section{PRELIMINARY FIELD TRIALS}

We tested a prototype of the TRICLOBS realtime night vision system in some nocturnal data collection trials in the field.

Figure 10 shows a typical example of a TRICLOBS image of a park scene registered at night in full darkness. Figs. 10a-c show respectively the three individual bands of the TRICLOBS image, i.e. the visual (wavelengths below $700 \mathrm{~nm}$ ), NIR (wavelengths between 700 and $900 \mathrm{~nm}$ ), and thermal $(8-14 \mu \mathrm{m}$ ) bands. Fig. 10d shows the actual false-color TRICLOBS image, obtained after the application of our new Color-the-Night remapping technique to the raw false color image that was initially formed by assigning the images Fig. 10a-c to each of the individual bands of a false color RGB image. Note that the resulting false color nightvision image closely resembles a corresponding daytime photograph of the same scene (Fig. 10e). Also, note that it is much easier to distinguish different materials and objects in Fig.9d, than in each of the individual bands (Fig. 10a-c).

\section{CONCLUSIONS}

In this paper we presented the prototype TRICLOBS portable tri-band realtime night vision system that can be used to demonstrate the operational value of a newly developed real-time color mapping that applies natural daylight colors to multi-band night-time images. The TRICLOBS system provides real-time co-aligned visual, near-infrared and thermal images. These co-aligned images can either be stored on on-board harddisks, or they can be processed in real-time by a (notebook) computer. The real-time color mapping is implemented as a lookup table transform. The results of some preliminary field trials clearly demonstrate the benefits of this systems for surveillance, navigation and target detection tasks. The resulting false color nightvision images closely resemble daytime images, while thermal targets are clearly distinguishable. 


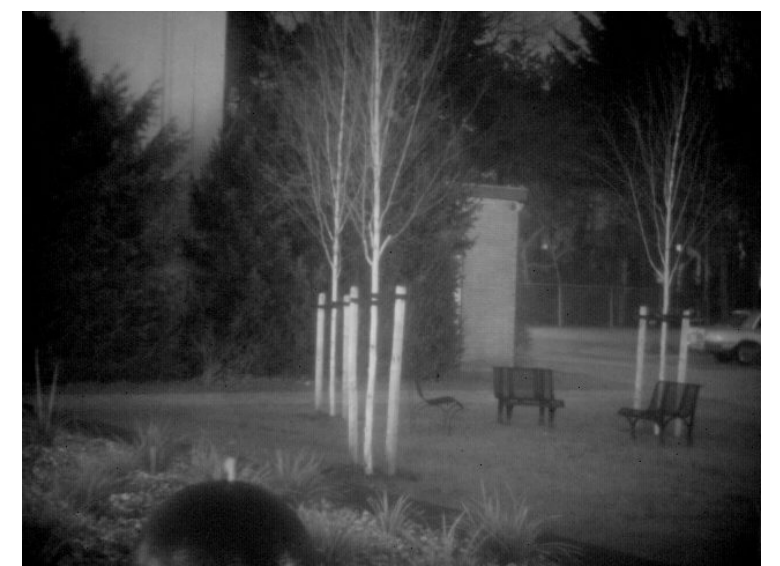

(a) Visual signal



(c) Thermal signal

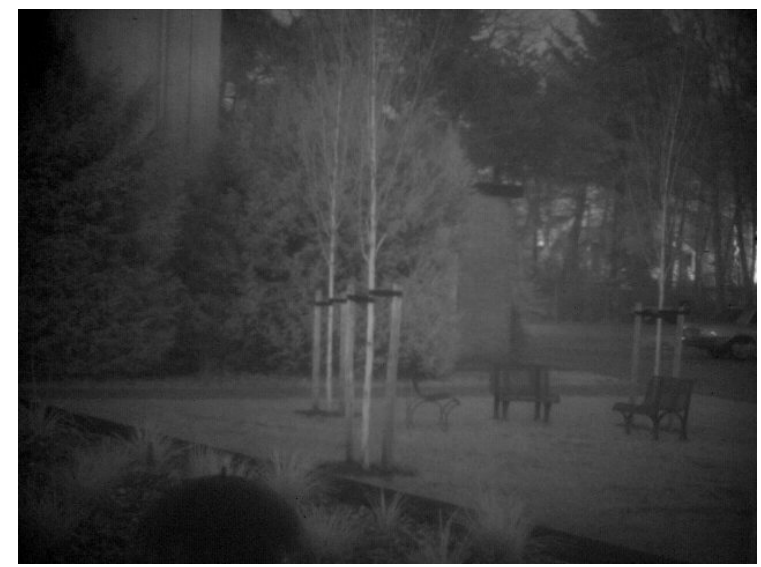

(b) NIR signal

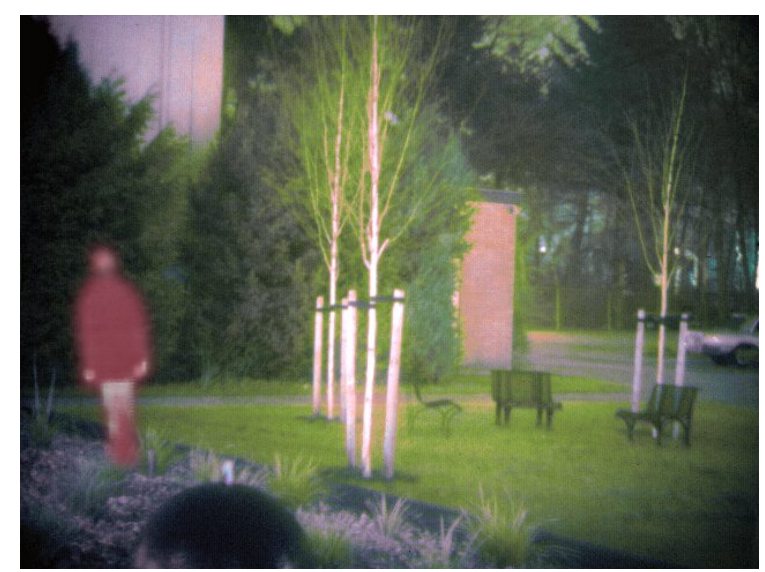

(d) Color fused result

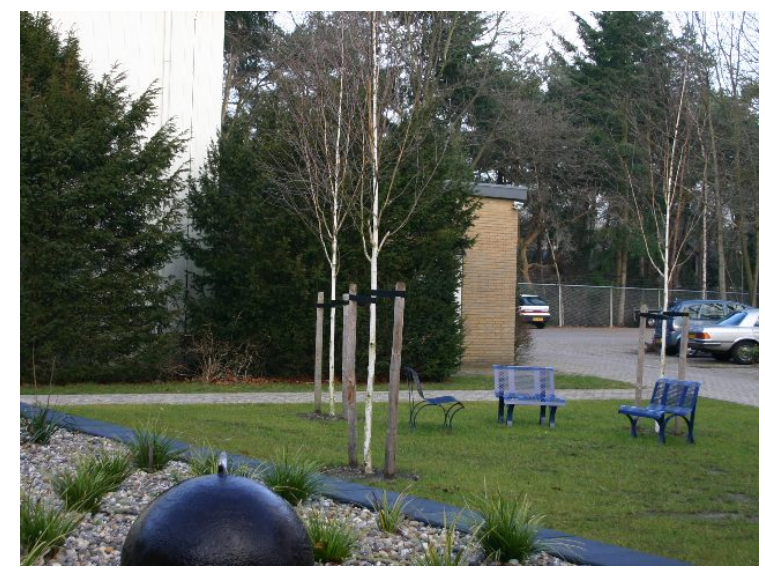

(e) Daytime color image (no target present)

Fig. 10 (a) Visual (wavelengths below $700 \mathrm{~nm}$ ), (b) NIR (wavelengths between 700 and $900 \mathrm{~nm}$ ), and thermal ( $8-14 \mu \mathrm{m}$ ) images of a park scene with a target person. (d) False color representation derived from (a-c) with the Color-the-Night mapping. (d) The result of our color remapping technique. (e) A daytime color photograph of the same scene. The square insets in images (c) and (d) represent their corresponding color tables. 


\section{REFERENCES}

1. Fay, D.A., Waxman, A.M., Aguilar, M., Ireland, D.B., Racamato, J.P., Ross, W.D., Streilein, W. and Braun, M.I., Fusion of multi-sensor imagery for night vision: color visualization, target learning and search, In: Proceedings of the $3^{\text {rd }}$ International Conference on Information Fusion, pp. TuD3-3-TuD3-10, ONERA, Paris, France, 2000.

2. Hogervorst, M.A. and Toet, A., Nighttime imagery in natural daytime colors, In: B.V. Dasarathy (Ed.), Multisensor, Multisource Information Fusion: Architectures, Algorithms, and Applications 2008, pp. The International Society for Optical Engineering, Bellingham, WA, USA, 2008.

3. Hogervorst,M.A., Toet,A., \& Kooi,F.L. (2006). TNO Defense Security and Safety. Method and system for converting at least one first-spectrum image into a second-spectrum image. 06076532 8-2202,

4. Howard, J.G., Warren, P., Klien, R., Schuler, J., Satyshur, M., Scribner, D. and Kruer, M.R., Real-time color fusion of E/O sensors with PC-based COTS hardware, In: W.R. Watkins, D. Clement \& W.R. Reynolds (Ed.), Targets and Backgrounds VI: Characterization, Visualization, and the Detection Process, pp. 41-48, The International Society for Optical Engineering, Bellingham, WA, 2000.

5. Li, G. and Wang, K., Applying daytime colors to nighttime imagery with an efficient color transfer method, In: J.G. Verly \& J.J. Guell (Ed.), Enhanced and Synthetic Vision 2007, pp. 65590L-1-65590L-12, The International Society for Optical Engineering, Bellingham, MA, 2007.

6. Ruderman, D.L., Cronin, T.W. and Chiao, C.-C., Statistics of cone responses to natural images: implications for visual coding, Journal of the Optical Society of America A, 15(8) ,pp. 2036-2045, 1998.

7. Shi, J., Jin, W., Wang, L. and Chen, H., Objective evaluation of color fusion of visual and IR imagery by measuring image contrast, In: H. Gong, Y. Cai \& J.-P. Chatard (Ed.), Infrared Components and Their Applications, pp. 594601, The International Society for Optical Engineering, Bellingham, MA, 2005.

8. Shi, J.-S., Jin, W.-Q. and Wang, L.-X., Study on perceptual evaluation of fused image quality for color night vision, Journal of Infrared and Millimeter Waves, 24(3),pp. 236-240, 2005.

9. Sinai, M.J., McCarley, J.S. and Krebs, W.K., Scene recognition with infra-red, low-light, and sensor fused imagery, In: Proceedings of the IRIS Specialty Groups on Passive Sensors, pp. 1-9, IRIS, Monterey, CA, 1999.

10. Sun, S., Jing, Z., Li, Z. and Liu, G., Color fusion of SAR and FLIR images using a natural color transfer technique, Chinese Optics Letters, 3(4) ,pp. 202-204, 2005.

11. Toet, A., Natural colour mapping for multiband nightvision imagery, Information Fusion, 4(3) ,pp. 155-166, 2003.

12. Tsagiris, V. and Anastassopoulos, V., Fusion of visible and infrared imagery for night color vision, Displays, 26(45) ,pp. 191-196, 2005.

13. Wang, L., Jin, W., Gao, Z. and Liu, G., Color fusion schemes for low-light CCD and infrared images of different properties, In: L. Zhou, C.-S. Li \& Y. Suzuki (Ed.), Electronic Imaging and Multimedia Technology III, pp. 459466, The International Society for Optical Engineering, Bellingham, WA, 2002.

14. Wang, L., Zhao, Y., Jin, W., Shi, S. and Wang, S., Real-time color transfer system for low-light level visible and infrared images in YUV color space, In: I. Kadar (Ed.), Signal Processing, Sensor Fusion, and Target Recognition XVI, pp. 1-8, The International Society for Optical Engineering, Bellingham, WA, 2007.

15. Waxman, A.M., et al., Solid-state color night vision: fusion of low-light visible and thermal infrared imagery, MIT Lincoln Laboratory Journal, 11 ,pp. 41-60, 1999.

16. Waxman, A.M., Fay, D.A., Gove, A.N., Seibert, M.C., Racamato, J.P., Carrick, J.E. and Savoye, E.D., Color night vision: fusion of intensified visible and thermal IR imagery, In: J.G. Verly (Ed.), Synthetic Vision for Vehicle Guidance and Control, pp. 58-68, The International Society for Optical Engineering, Bellingham, WA, 1995.

17. Yue, Z. and Topiwala, P., Real-time EO/IR sensor fusion on a portable computer and head-mounted display, In: B.V. Dasarathy (Ed.), Multisensor, Multisource Information Fusion: Architectures, Algorithms, and Applications 2007, pp. 657106- The International Society for Optical Engineering, Bellingham, WA, 2007.

18. Zheng, Y., Hansen, B.C., Haun, A.M. and Essock, E.A., Coloring night-vision imagery with statistical properties of natural colors by using image segmentation and histogram matching, In: R. Eschbach \& G.G. Marcu (Ed.), Color imaging X: processing, hardcopy and applications, pp. 107-117, The International Society for Optical Engineering, Bellingham, WA, 2005. 